\section{Effects of antenatal stress and anxiety}

\author{
Implications for development and psychiatry
}

VIVETTE GLOVER and THOMAS G. O'CONNOR bio-social mechanisms of risk and resilience, and develop the most effective treatments.

\section{EVIDENCE FROM STUDIES IN HUMANS}

The studies of the effects of antenatal stress and anxiety in humans have concentrated on obstetric outcome. Hansen et al (2000) found that women who experienced severe life events in the first trimester of pregnancy had a $50 \%$ increase in the rate of congenital abnormalities in cranial-neural crestderived organs (e.g. cleft palate). Even greater risk was associated with the most severe and rare stress, the unexpected death of a child.

Preterm labour and low birthweight for gestational age are the outcomes linked most consistently with antenatal stress or anxiety in humans (Hedegaard et al, 1993; Lou et al, 1994). These findings are relatively robust across different measures of stress/anxiety. In one study of 8719 women, Hedegaard et al (1993) reported a significant association between selfreported general distress at 30 weeks and an increased risk of preterm delivery, defined as $<37$ weeks (relative risk was found to be 1.22 for moderate and 1.75 for high distress $v$. low distress); however, the effect of distress early in pregnancy on obstetric outcome was not significant. Lou et al (1992) have shown that individuals who experienced severe stressful events during pregnancy showed a $50 \%$ increase in marked premature delivery (babies born at $<34$ weeks).

Consistent with the animal evidence, some early studies in humans have shown links between antenatal stress or anxiety and behavioural/emotional disturbance in the child. However, the strength of this association was uncertain as these studies did not covary antenatal risks, such as smoking, and failed to distinguish between ante- and postnatal stress. In next month's issue of the Journal we will be reporting the strongest evidence to date for such an association using a large community sample, the Avon Longitudinal Study of Parents and Children (ALSPAC) cohort (O’Connor et al, 2002). There was a strong link between maternal anxiety in the third trimester and behavioural/emotional problems in the resulting children at 4 years. This link was maintained after 
controlling for maternal anxiety on four successive postnatal periods, up to 33 months. After controlling for antenatal, obstetric and socio-economic risks, antenatal and postnatal depression and multiple indicators of postnatal anxiety, we found that self-reported antenatal anxiety at 32 weeks' gestation predicted severe behavioural/emotional problems in both boys and girls. Hyperactivity/inattention in boys was the one sub-scale that remained significantly associated with anxiety in late gestation after controlling for the above covariates. The findings would not be easily explicable by genetic transmission or anxious parenting, because postnatal anxiety on multiple subsequent occasions was statistically controlled for. Of particular interest was the finding that the effect derived from antenatal anxiety and not depression, despite the marked overlap between these dimensions. Anxiety, rather than depression, is the analogue of the risk used in animal studies.

Analyses from the ALSPAC cohort also found evidence of a link between antenatal anxiety and neurological development. High maternal anxiety at 18 weeks' gestation predicted atypical laterality (i.e. mixed handedness) in the child (further details available from the author upon request), independently of maternal and paternal handedness and obstetric and other antenatal risks. No association was found with postnatal anxiety, indicating that maternal anxiety had qualitatively different effects on child handedness in the antenatal and postnatal periods. An association between antenatal anxiety and mixed handedness in the child was also found in a Danish cohort (C. Obel et al, personal communication, 2002). If antenatal anxiety is causally associated with mixed handedness, then it could have a role in other disorders associated with mixed handedness that have a neurodevelopmental component, such as dyslexia and autism.

Several plausible mechanisms linking antenatal stress/anxiety and disturbances in offspring have been suggested. Antenatal anxiety could increase the risk for offspring disturbance by shortening gestation and reducing birthweight. Preterm birth is the single largest perinatal risk factor for later morbidity, including attention-deficit hyperactivity disorder and schizophrenia, and being small for gestational age is associated with similar adverse mental and behavioural problems (Hultman et al, 1999).

VIVETTE GLOVER, DSc, Institute of Reproductive and Developmental Biology, Imperial College London; THOMAS G. O'CONNOR, PhD, Departments of Child \& Adolescent Psychiatry and Social Genetic and Developmental Psychiatry Research Centre, Institute of Psychiatry, London, UK

Correspondence: Professor Vivette Glover, Fetal and Neonatal Stress Research Group, Institute of Reproductive and Developmental Biology, Faculty of Medicine, Imperial College, Hammersmith Campus, Du Cane Road, London WI2 ONN, UK. Tel: 0207594 2136; Fax: 0207594 2138; e-mail: v.glover@ic.ac.uk

(First received 2 January 2002, accepted 22 January 2002)

Research supporting a mechanism involving the HPA axis is increasing. Gitau et al (2001) found that maternal and foetal levels of the stress hormone cortisol were correlated $(r=0.58)$, suggesting that sufficient maternal cortisol can cross the placenta to significantly alter foetal exposure. Other research indicated that elevated maternal anxiety in late (but not early) pregnancy is associated with impaired blood flow or raised resistance index to the foetus through the maternal uterine arteries (Teixeira et al, 1999). High resistance is associated with adverse obstetric outcome, particularly intra-uterine growth restriction and pre-eclampsia, and could therefore also help to explain why anxious mothers have babies that are small for their gestational age. It is unlikely that the same mechanism explains all obstetric and behavioural/ emotional/neurodevelopmental outcomes. Further research on the HPA axis as a causal mechanism is required and will need to take into account the fact that pregnancy itself alters the functioning of the HPA axis.

Different effects of antenatal mood are observed at different stages of gestation because the foetus shows different vulnerabilities depending on its state of development. Thus, severe stress experienced in early gestation, when the organs are being formed, affects physical outcomes such as cleft palate. In contrast, the effects of stress/anxiety later in pregnancy, when neuronal connections are being made in the brain, are on behavioural/emotional outcomes. However, questions about developmental timing persist and this remains a central area for research.

\section{IMPLICATIONS FOR IDENTIFYING DEVELOPMENTAL MECHANISMS}

In conclusion, we consider examples of how studies of antenatal stress/anxiety could challenge current concepts of development and psychiatric risk. The first example concerns the role of 'obstetric factors' (e.g. low birthweight), which have been given considerable attention in psychiatric research. The role of antenatal anxiety is considered rarely in these studies and, as the preceding discussion suggests, poor obstetric outcome might not only be a causal agent for later morbidity in the offspring but also an outcome of antenatal stress or affective disorder. A second misspecification that can result from ignoring antenatal maternal mood is in the area of postnatal depression. Frequently, postnatal depression is preceded by antenatal depression (Evans et al, 2001) as well as antenatal anxiety; it could be that some of the effects on the child attributed to postnatal depression may derive instead from antenatal mood, perhaps especially anxiety. Third, the possibility that early stress exposure increases the risk for child and adult behavioural/emotional disturbances associated with the HPA axis (e.g. depression) provides an important complementary or perhaps even alternative - hypothesis to those derived from contemporary genetic and psychosocial research. Given its obvious scientific, clinical and policy implications for psychology and psychiatry, the hypothesis that antenatal stress or anxiety in humans affects developmental programming in a way that leads to later psychopathology requires systematic and direct testing.

\section{DECLARATION OF INTEREST}

None.

\section{REFERENCES}

Caldji, C., Diorio, J. \& Meaney, M. J. (2000) Variations in maternal care in infancy regulate the development of stress reactivity. Biological Psychiatry, 48, I164-1174. 
Evans, J., Heron, J., Francomb, J., et al (200I) Cohort study of depressed mood during pregnancy and after childbirth. BMJ, 323, 257-260.

Gitau, R., Fisk, N., Teixeira, J., et al (200I) Fetal HPA stress responses to invasive procedures are independent of maternal responses. Journal of Clinical and Endocrinological Metabolism, 86, 104-109.

Hansen, D., Lou, H. C. \& Olsen, J. (2000) Serious life events and congenital malformations: a national study with complete follow-up. Lancet, 356, 875-880.

Hedegaard, M., Henriksen, T. B., Sabroe, S., et a (1993) Psychological distress in pregnancy and preterm delivery. BMJ, 307, 235-239.
Hultman, C. M., Sparen, P., Takei, N., et al (1999) Prenatal and perinatal risk factors for schizophrenia, affective psychosis, and reactive psychosis of early onset: case-control study. BMJ, 318, 42I-426.

Lou, H. C., Nordentoft, M., Jensen, F., et al (1992) Psychosocial stress and severe prematurity. Lancet, 340, 54.

_, Hansen, D., Nordentoft, M., et al (1994) Prenata stressors of human life affect fetal brain development. Developmental Medicine and Child Neurology, 36, 826-832.

O'Connor, T. G., Heron, J., Golding, J., et al (2002) Maternal antenatal anxiety and children's behavioural/ emotional problems at 4 years. Report from the Avon
Longitudinal Study of Parents and Children. British Journal of Psychiatry, in press.

Schneider, M., Moore, C. F., Roberts, A. D., et a (200I) Prenatal stress alters early neurobehaviour, stress reactivity and learning in non-human primates: a brief review. Stress, 4, 183-193.

Teixeira, J. M., Fisk, N. M. \& Glover, V. (1999) Association between maternal anxiety in pregnancy and increased uterine artery resistance index: cohortbased study. BMJ, 318, 153-157.

Weinstock, M. (200I) Alterations induced by gestational stress in brain morphology and behaviour of the offspring. Progress in Neurobiology, 65, 427-45I. 